\title{
Avaliação biológica e econômica do uso de flunixin meglumine em vacas e novilhas de corte inseminadas em tempo fixo
}

\author{
Luiz Francisco Machado Pfeifer ${ }^{1}$, Augusto Schneider ${ }^{2}$, José Wilson da Silva Neto ${ }^{2}$, Evâneo \\ Alcides Ziguer ${ }^{2}$, Nelson José Laurino Dionello ${ }^{1}$, Marcio Nunes Corrêa ${ }^{2}$
}

\footnotetext{
${ }^{1}$ Faculdade de Agronomia, Departamento de Zootecnia, Universidade Federal de Pelotas, CEP: 96010-900, Pelotas/RS.

2 Faculdade de Veterinária, Departamento de Clínicas Veterinária, Universidade Federal de Pelotas, CEP: 96010-900, Pelotas/RS.
}

\begin{abstract}
RESUMO - Objetivou-se verificar a redução de perdas embrionárias por meio do bloqueio da secreção de prostaglandina com a utilização de flunixin meglumine (FM) e avaliar o retorno econômico desta técnica. No experimento 1, utilizaram-se vacas de corte no pós-parto e, no experimento 2, somente novilhas. Todas as fêmeas foram sincronizadas e inseminadas em tempo fixo (IATF) e, após 14 dias da IATF, foram distribuídas em dois grupos: grupo flunixin meglumine (GFM), composto de 59 vacas no experimento 1 e 23 novilhas no experimento 2; e grupo controle (GC), de 56 vacas no experimento 2 e 20 novilhas no experimento 1 , que receberam ou não flunixin no dia 14 , respectivamente. $O$ tratamento com flunixin não influenciou a taxa de prenhez, que, no experimento 1 , foi de $42,4 \%$ no grupo flunixin e $42,8 \%$ no grupo controle e, no experimento 2, de $39,1 \%$ no grupo flunixin e $25,0 \%$ no grupo controle. Verificou-se rentabilidade de 21,$62 ; 9,24 ; 14,36$ e $12,06 \%$ para os protocolos dos grupos controle e flunixin dos experimentos 1 e 2, respectivamente. A taxa de prenhez não foi influenciada pelo uso de flunixin meglumine no dia 14 após inseminação artificial em vacas no pós-parto e novilhas inseminadas em tempo fixo.
\end{abstract}

Palavras-chave: antiinflamatório não-esteróide, bovinos de corte, mortalidade embrionária

\section{Biological and economic evaluation of flunixin meglumine in postpartum beef cows and heifers inseminated at fixed time}

\begin{abstract}
This study aimed to evaluate the effectiveness and the economical advantage of using flunixin meglumine (FM) to block uterine secretion of prostaglandin in order to reduce embryonic losses in beef cattle females. Postpartum cows (E1) and heifers (E2) were submitted to estrus synchronization and inseminated at fixed time. Fourteen days after the insemination the animals were assigned to the FM treatment group (FMG, including 59 cows and 23 heifers) and to the control group (CG, including 56 cows and 20 heifers). Pregnancy rates (42.4\% for FMG and $42.8 \%$ for CG in E1) and (39.1\% for FMG and $25.0 \%$ for CG in E2) did not differ between groups. Estimates of net income were 21.62, 9.24, 14.36 and $12.06 \%$ for the CG e FMG protocols in cows and in heifers, respectively. The use of FM at 14 days after AI did not affect pregnancy rates of postpartum beef cows and heifers.
\end{abstract}

Key Words: anti-inflammatory, beef cattle, embryonic mortality

\section{Introdução}

A lucratividade na pecuária leiteira e de corte é resultado da eficiência reprodutiva, que influencia a produção de leite e o número de bezerros nascidos. A perda embrionária é a principal causa da baixa eficiência reprodutiva em bovinos (Zavy et al., 1994; López-Gatius et al., 1996; Hansen, 2002). Durante o ciclo estral nos bovinos, a luteólise ocorre em torno do $16^{\mathrm{O}}$ dia pós-ovulação (Gonçalves et al., 2001), em virtude da secreção pulsátil de prostaglandina $\left(\mathrm{PGF}_{2} \alpha\right)$ pelo endométrio (Peterson et al., 1975; Kindhal et al., 1976). O mecanismo endócrino que controla a liberação endometrial da $\mathrm{PGF}_{2} \alpha$ ainda não foi totalmente elucidado.

O reconhecimento e estabelecimento da gestação envolvem várias interações entre o concepto, o útero e o corpo lúteo (Arosh et al., 2004) e, para isso, o concepto bovino secreta interferon-tau (IFN- $\tau$ ) - com pico de secreção entre o $17 \underline{0}$ e o $22^{\circ}$ dia de gestação - inibindo a liberação endometrial de $\mathrm{PGF}_{2} \alpha$ (Kerbler et al., 1997).

Há evidências de que o uso de medicamentos que reduzem a síntese de $\mathrm{PGF}_{2} \alpha$ pelo endométrio, por meio da inibição da enzima ciclo-oxigenase na cascata do ácido araquidônico (Odensvik et al., 1989), pode favorecer a 
manutenção do corpo lúteo no período crítico de reconhecimento materno da gestação (Binelli et al., 2001). Odensvik et al. (1998) observaram que a administração oral de flunixin meglumine (FM), um antiinflamatório não-esteróide, retarda a luteólise, aumentando a duração do ciclo estral. Acredita-se que este atraso permite ao concepto se desenvolver e produzir IFN- $\tau$ suficiente para prevenir a luteólise e manter a gestação (Aké-López et al., 2005).

A maioria das pesquisas desenvolvidas como alternativas para o aumento da produtividade pecuária não apresenta nem discute a economicidade da aplicação das novas propostas biológicas estudadas; restringe-se aos fatores técnicos e desconsidera o impacto econômico gerado pelos resultados observados (Pötter et al., 2000). Neste âmbito, a modelagem e a simulação têm sido utilizadas para permitir este tipo de estudo com a vantagem de acarretar baixo custo e reduzido tempo de avaliação (Naazie et al., 1999).

Objetivou-se com este estudo avaliar a influência da aplicação de flunixin meglumine no momento do reconhecimento da gestação sobre a taxa de prenhez de vacas de corte no pós-parto e de novilhas submetidas a inseminação artificial em tempo fixo (IATF) e estimar o impacto econômico desta técnica.

\section{Material e Métodos}

No experimento 1, foram utilizadas 115 vacas mestiças (Bos taurus $\times$ Bos indicus) aproximadamente aos 100 dias pós-parto, mantidas a pasto em campo nativo, no município de Dom Pedrito - RS. Foram selecionadas vacas com condição corporal (CC) de 2,5 (em escala de 1 a 5, em que 1 é muito magra e 5 obesa). As vacas foram submetidas a exame ginecológico e todas apresentavam atividade ovariana no início dos tratamentos hormonais.

Todas as fêmeas receberam um pessário intravaginal de esponja impregnado com $250 \mathrm{mg}$ de acetato de medróxiprogesterona - MAP (Galena Química e Farmacêutica Ltda), e realizou-se aplicação intramuscular de 2,5 mg de benzoato de estradiol - BE (DEGLtda), no dia 0 (D0). A remoção do pessário foi realizada no dia 8 (D8), simultaneamente à aplicação de $250 \mu \mathrm{g}$ de cloprostenol sódico (Ciosin $\AA$, Schering-Plough Saúde Animal Indústria e Comércio Ltda) (meia dose, $500 \mu \mathrm{g} / 2 \mathrm{~mL}$ ). Após 54 horas da retirada dos pessários, procedeu-se à aplicação de $50 \mu \mathrm{g}$ de acetato de fertirelina (Fertigen ${ }^{\circledR}$, Schering-Plough Saúde Animal Indústria e Comércio Ltda) (meia dose, $100 \mu \mathrm{g} / 2 \mathrm{~mL}$ ) e à inseminação artificial (Figura 1).

Aos 14 dias após a inseminação, os animais foram divididos em dois grupos: 59 foram alocados no grupo flunixin meglumine (GFM) e receberam aplicação intramuscular de $11 \mathrm{mg}$ de flunixin meglumine (Banamine ${ }^{\circledR}$, ScheringPlough Saúde Animal Indústria e Comércio Ltda) (1,1 mg/mL); e 56 foram alocados no grupo controle (GC), sem aplicação de flunixin meglumine (Figura 1).

Com a retirada dos pessários intravaginais (D8), foi realizado o desmame dos bezerros até o momento da IATF (D10).

No experimento 2, realizado sob mesmas condições do experimento 1 , foram utilizadas 43 novilhas mestiças (Bos taurus $\times$ Bos indicus) com atividade ovariana ao exame ginecológico. Todas as fêmeas receberam pessário intravaginal de esponja impregnado com $250 \mathrm{mg}$ de MAP e realizou-se aplicação intramuscular de $2,5 \mathrm{mg}$ de benzoato de estradiol no dia 0 . A remoção do pessário foi realizada no dia 8, comcomitantemente à aplicação de $250 \mu \mathrm{g}$ de

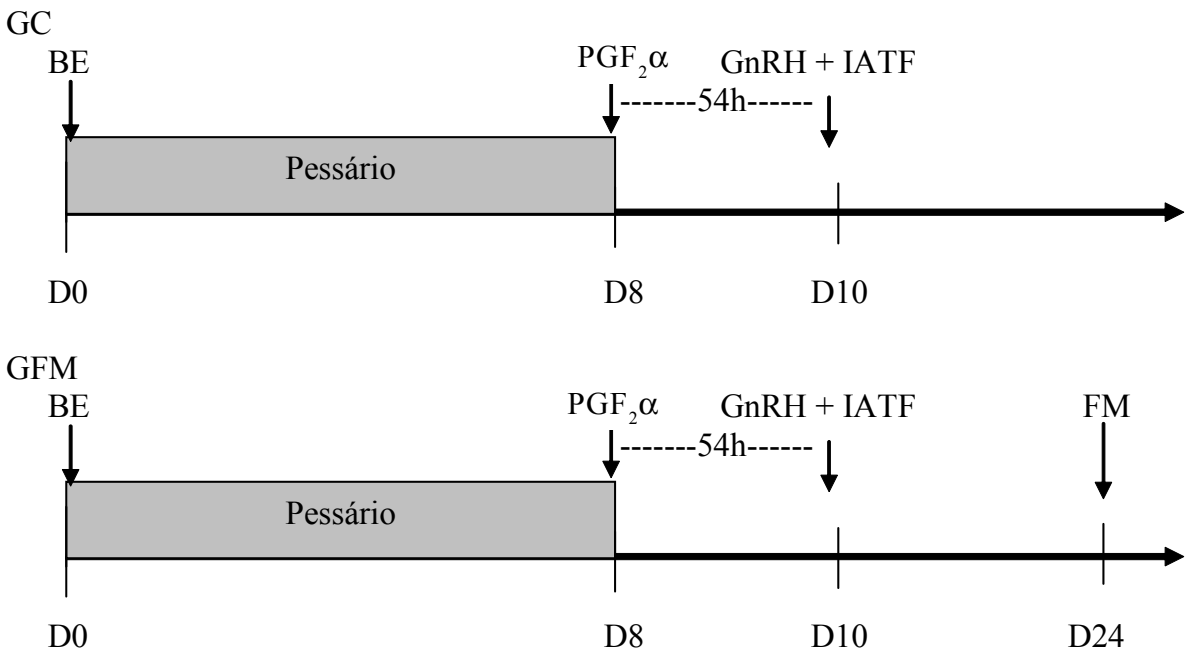

Figura 1 - Protocolos para sincronização de estros utilizados no experimento 1 para o Grupo Flunixin Meglumine (GFM) e o Grupo Controle (GC). 


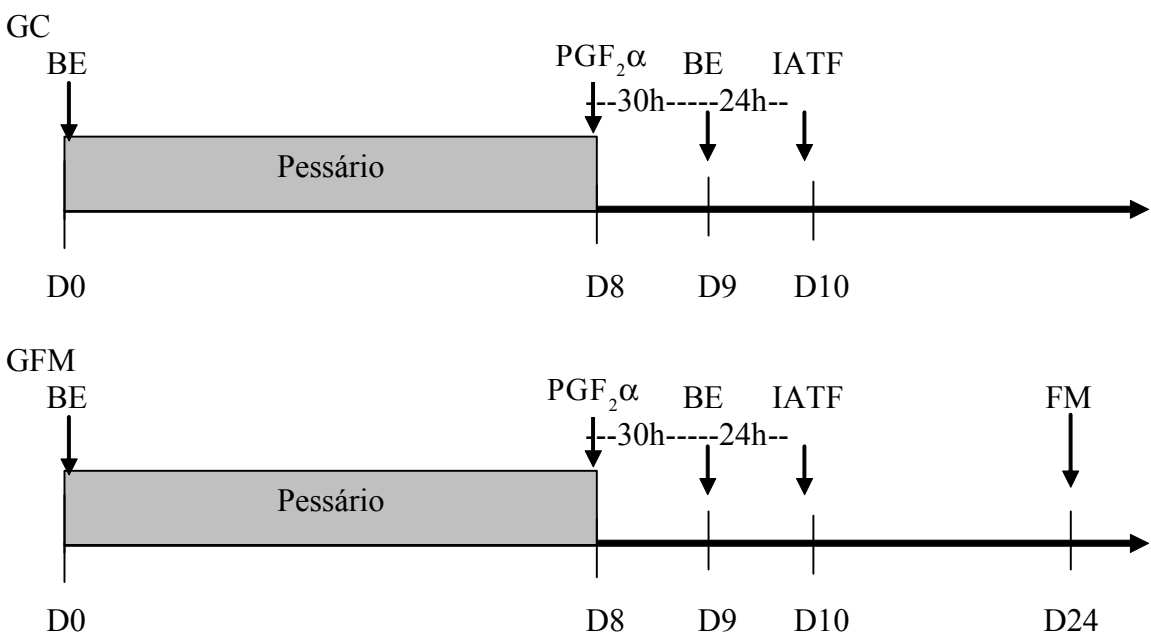

Figura 2 - Protocolos para sincronização de estros utilizados no experimento 2 para o Grupo Flunixin Meglumine (GFM) e o Grupo Controle (GC).

cloprostenol sódico (meia dose, $500 \mu \mathrm{g} / 2 \mathrm{~mL}$ ). Trinta horas após a retirada dos pessários, realizou-se a aplicação de 0,5 mg de benzoato de estradiol para indução da ovulação. Após 24 horas da aplicação do benzoato de estradiol, procedeu-se à inseminação artificial em tempo fixo (Figura 2).

Após 14 dias da inseminação artificial, dividiram-se os animais em dois grupos: 23 animais foram alocados no grupo GFM e receberam aplicação intramuscular de $11 \mathrm{mg}$ de flunixin meglumine $(1,1 \mathrm{mg} / \mathrm{mL})$; e 20 animais foram alocados no grupo controle (GC) e não receberam nenhum tratamento (Figura 2).

O diagnóstico de gestação foi realizado 30 dias após a inseminação artificial por meio de ultra-sonografia em equipamento Piemedical Answer com sonda setorial convexa de $5 \mathrm{MHz}$.

A avaliação econômica foi realizada para os animais dos experimentos $1 \mathrm{e} 2$, considerando individualmente cada tratamento. $O$ custo operacional referente à implantação de cada técnica de sincronização foi obtido considerando os custos com protocolos hormonais, as doses de sêmen, o material descartável utilizado para a inseminação artificial, a remuneração do inseminador e o custo adicional do flunixin meglumine. Nos custos com materiais utilizados na inseminação artificial, incluíram-se luvas de palpação retal, bainhas do aplicador e papel-toalha. Na variável remuneração do inseminador, considerou-se como custo $1 \mathrm{~kg}$ de boi por fêmea inseminada (Tabela 1). As receitas foram calculadas pelo total de quilos de bezerros desmamados utilizando-se as taxas de prenhez obtidas nos dois grupos em ambos os experimentos. Consideraram-se peso médio ao nascimento de $34 \mathrm{~kg}$ (Lobato et al., 1998), taxa de ganho médio diário (GMD) de $0,67 \mathrm{~kg}$ (Lobato et al., 1998), índice de mortalidade de 6\% (Corrêa et al., 2000), desmame aos 210 dias e valor de venda do bezerro de $\mathrm{R} \$ 1,70 / \mathrm{kg}$.

A partir do banco de dados, foram gerados os cálculos de custos e receitas e a simulação econômica para os grupos. A rentabilidade de cada protocolo foi calculada utilizando-se também a função financeira taxa interna de retorno (TIR) e considerando-se para base de cálculo as receitas líquidas totais e o período de 15 meses, referente

Tabela 1 - Custo individual de implantação da técnica de acordo com o protocolo utilizado e a representatividade de cada variável

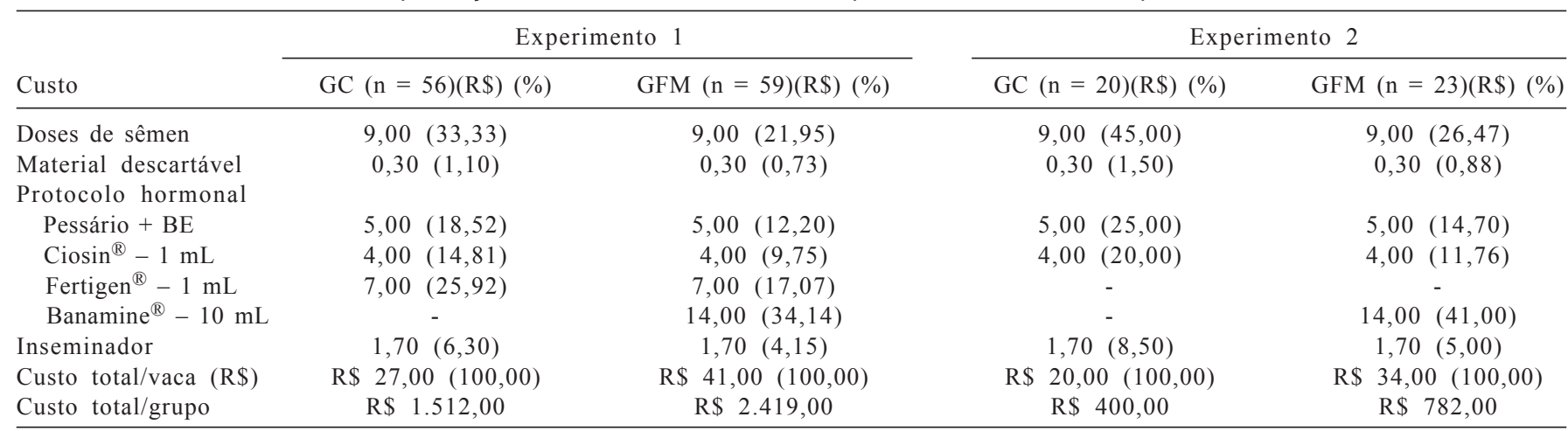


ao tempo necessário para gestação e desmame, ou seja, tempo para geração de receita e retorno do capital investido. Os dados referentes à taxa de prenhez foram avaliados pelo teste do qui-quadrado, por meio do programa Statistix ${ }^{\circledR}$ (2004).

\section{Resultados e Discussão}

No experimento 1, a taxa média de prenhez foi de $42,6 \%$ (49/115) e, quando considerados os tratamentos separadamente, foi de 42,4\% (25/59) para o grupo com aplicação de flunixin e e 42,8\% (24/56) para o grupo controle, que não diferiram entre $\mathrm{si}(\mathrm{P}>0,05)$. No experimento 2 , a taxa média de prenhez foi de $31,8 \%(14 / 44)$ e, quando considerados os grupos separadamente, de $39,1 \%(9 / 23)$ para o grupo flunixin e $25,0 \%(5 / 20)$ para o grupo controle, que também não diferiram entre si $(\mathrm{P}=0,27)$ (Tabela 2$)$.

Os resultados da taxa média de prenhez obtidos no experimento 1 corroboram os verificados por Castilho \& Pfeifer (2005), que, utilizando esponjas impregnadas com MAP em vacas de corte no pós-parto, obtiveram $42,4 \%$ de taxa de prenhez. Os resultados encontrados no experimento 2 , no entanto, foram inferiores à média observada por Colazo et al. (2006), que verificaram taxa de prenhez de 57\% em novilhas de corte submetidas a protocolo semelhante com aplicação de progesterona.

A diferença no índice de prenhez verificada entre o grupo tratado com antiinflamatório e o grupo controle dos experimentos 1 e 2 difere dos resultados encontrados por Merril et al. (2003), que observaram tendência $(P=0,17)$ de maior taxa de prenhez em vacas tratadas com flunixin meglumine no dia 14 após inseminação em comparação a vacas sem nenhum tratamento. Entretanto, os animais utilizados por Merril et al. (2003) foram induzidos ao estresse, o que pode ter influenciado a resposta ao antiinflamatório, visto que manejos estressantes após inseminação artificial podem induzir a reabsorção embrionária (Hansen et al., 2002). Além disso, outros experimentos (Odensvik et al., 1998; Aba et al., 2000; Aké-López et al., 2005) comprovaram aumento no intervalo interestral e maior intervalo ovulaçãoluteólise. Portanto, maior desenvolvimento embrionário

Tabela 2 - Taxa de prenhez nos grupos GC e GFM dos experimentos 1 e 2

\begin{tabular}{lccc}
\hline Experimento & \multicolumn{3}{c}{ Taxa de prenhez } \\
\cline { 2 - 4 } & Total $(\%)$ & GC $(\%)$ & GFM $(\%)$ \\
\hline E1 & 42,6 & 42,8 & 42,4 \\
E2 & 31,8 & 25,0 & 39,1 \\
\hline
\end{tabular}

poderia ocorrer como conseqüência do prolongamento do ciclo estral, aumentando a secreção de IFN- $\tau$ a um nível suficiente para induzir o reconhecimento da gestação (Aké-López et al., 2005). Como neste experimento não foi realizada observação de estro após a inseminação, não é possível inferir se houve prolongamento do ciclo e se esse possível prolongamento teve alguma correlação com a taxa de prenhez.

Apesar de este trabalho ter sido realizado com o objetivo de relacionar o resultado da concepção à inibição da $\mathrm{PGF}_{2} \alpha$ no momento do reconhecimento da gestação, não é possível descartar outros fatores que contribuem diretamente para a manutenção da gestação, como a concentração sérica de progesterona, que possui correlação positiva com o reconhecimento da gestação (Bullman \& Lamming, 1978; Lamming et al., 1989; Mann et al., 1999) e com o aumento do desenvolvimento embrionário (Garret et al., 1988). Vacas submetidas à inseminação por tempo fixo são mais predispostas a fases luteais de curta duração, pois a ovulação induzida muitas vezes não permite que o útero tenha exposição adequada ao estrogênio no período pré-ovulatório, resultando em aumento da responsividade uterina ao estímulo da ocitocina e em maior liberação de $\mathrm{PGF}_{2} \alpha$ e luteólise e, conseqüentemente, maior reabsorção embrionária (Mann \& Lamming, 2000).

Apesar de o concepto secretar quantidades razoáveis de IFN- $\tau$ no dia 14 , alguns autores sugerem que os pulsos de $\mathrm{PGF}_{2} \alpha$ só atingem seu ponto máximo entre o 150 e o 170 dia do ciclo estral (Binelli et al., 2001; McCraken et al., 1999; Thatcher et al., 1986). Como a meia-vida do flunixin meglumine é de 24 horas (Guilbault et al., 1987), é possível que esse período seja o mais apropriado para aplicação visando à inibição dos efeitos da $\mathrm{PGF}_{2} \alpha$ no corpo lúteo. Neste trabalho, o momento de aplicação do flunixin pode não ter sido o de maior eficácia. Merril et al. (2003), no entanto, encontraram resultados expressivos com a aplicação no dia 14 após a inseminação artificial. Assim, para elucidar os eventos relacionados à concepção e melhorar a taxa de prenhez com o uso de fluxinin meglumine após a concepção, são necessários mais estudos relacionados ao melhor momento da aplicação, à dose e ao tempo de uso deste medicamento.

Considerando os custos totais de implantação (Tabela 1), observou-se um custo superior da implantação do GFM, de $51,85 \%$ no experimento 1 e de $70,00 \%$ no experimento 2 , em decorrência da utilização do flunixin meglumine, que representou 34,14 e $41,00 \%$ dos custos totais nos experimentos 1 e 2, respectivamente. Quando considerado o número de bezerros nascidos, os custos de implantação para o expe- 
Tabela 3 - Total (em $\mathrm{kg}$ ) de bezerros desmamados e da receita bruta em cada grupo

\begin{tabular}{lccccc}
\hline & \multicolumn{2}{c}{ Experimento 1} & & \multicolumn{2}{c}{ Experimento 2 } \\
\cline { 2 - 3 } \cline { 6 - 7 } Variável & GC & GFM & & GC & GFM \\
\hline № bezerros desmamados* & 22,53 & 23,52 & & 4,7 & 8,45 \\
Peso ao nascimento $(\mathrm{kg})$ & 34,00 & 34,00 & & 34,00 & 34,00 \\
GMD (kg) & 0,67 & 0,67 & & 0,67 & 0,67 \\
Peso à desmama (kg) & 174,70 & 174,70 & & 174,70 & 174,70 \\
Total (kg bezerros) & $3.935,99$ & $4.108,94$ & & 820,15 & $1.476,22$ \\
Receita bruta & $6.691,18$ & $6.985,20$ & & $1.394,26$ & $2.509,57$ \\
total (R\$) & & & & & \\
\end{tabular}

* Considerando o índice de mortalidade de 6\%.

rimento 1 foram de $\mathrm{R} \$ 67,11$ e R $\$ 102,85 /$ bezerro nascido nos grupos controle e com aplicação de flunixin meglumine, respectivamente. No experimento 2 , o custo foi de $\mathrm{R} \$ 85,10$ e R \$92,54/bezerro nascido nos grupos controle e com aplicação de fluxinin, respectivamente. Portanto, a necessidade de observação dessa variação no aspecto econômico das técnicas é importante, uma vez que a eficiência econômica é fundamental para manutenção de qualquer atividade pecuária (Ferreira et al., 2004).

Ao dividir o total arrecadado em reais pelo número de animais de cada grupo, a renda bruta (RB) por vaca no experimento 1 foi de $\mathrm{R} \$ 119,49$ e $\mathrm{R} \$ 118,39$ para os grupos controle e com flunixin, respectivamente, e, no experimento 2, de $\mathrm{R} \$ 69,71$ e $\mathrm{R} \$ 109,11$ para os grupos controle e flunixin, respectivamente.

A alteração na receita bruta entre os grupos foi bem mais expressiva no experimento 2 , em virtude da diferença $(14 \%)(\mathrm{P}=0,27)$ na taxa de prenhez, que apresentou acréscimo de 79,99\% na receita do grupo com aplicação de flunixin. A maior receita bruta neste grupo está associada à maior quantidade (em $\mathrm{kg})$ de bezerros desmamados, que, por sua vez, se relacionou à maior taxa de desmame.

A receita líquida (RL) foi de $\mathrm{R} \$ 5.179,18$ e $\mathrm{R} \$ 4.566,20$ no experimento 1 e de $\mathrm{R} \$ 994,26$ e $\mathrm{R} \$ 1.727,57$ no experimento 2 para os grupos controle e com aplicação de flunixin, respectivamente. Quando considerada a RL, foi registrada receita por animal de $\mathrm{R} \$ 92,48$; $\mathrm{R} \$ 77,39$; $\mathrm{R} \$ 49,71 ; \mathrm{R} \$ 75,11$ para os grupos controle e com flunixin dos experimentos 1 e 2 , respectivamente. A receita líquida por animal reflete as maiores taxas de desmame observadas no experimento 1 e a diferença $(P=0,27)$ entre os grupos controle e flunixin do experimento 2 , logo, aumentos na taxa de prenhez podem determinar maiores retornos líquidos para o produtor.

As taxas de rentabilidade para os protocolos dos grupos controle e com aplicação de flunixin foram de 21,62; 9,$24 ; 14,36$ e $12,06 \%$ nos experimentos 1 e 2 , respectiva- mente, e podem ser consideradas elevadas, pois foram incluídos somente os custos das técnicas implantadas. Entretanto, em ambos os grupos, os demais custos relacionados podem ser considerados fixos. Além disso, apesar de o grupo com aplicação de flunixin ter gerado maior receita líquida, os indicadores de rentabilidade deste grupo não apresentaram a mesma tendência, em virtude do maior investimento necessário para implantação deste protocolo de sincronização.

A diferença na taxa de prenhez obtida no experimento 2 pode ter sido comprometida pelo reduzido número de animais utilizados, pois análises estatísticas como o quiquadrado costumam detectar diferenças quando a amostra é grande.

\section{Conclusões}

A taxa de prenhez não foi influenciada pela aplicação de flunixin meglumine no 14을 dia após inseminação artificial em vacas no pós-parto e em novilhas inseminadas em tempo fixo.

\section{Literatura Citada}

ABA, M.A.; KINDAHL, H.; FORSBERG, M. et al. Levels of progesterone and changes in prostaglandin $\mathrm{F}_{2} \alpha$ release during luteolysis and early pregnancy in llamas and the effect of treatment with flunixin meglumine. Animal Reproduction Science, v.59, p.87-97, 2000.

AKÉ-LÓPEZ, R.; SEGURA-CORREA, J.C.; QUINTAL-FRANCO, J. Effect of flunixin meglumine on the corpus luteum and possible prevention of embryonic loss in Pelibuey ewes. Small Ruminant Research, v.59, p.83-87, 2005.

AROSH, A.; BANU, S.K.; KIMMINS, S. et al. Effect of interferon$t$ on prostaglandin biosynthesis, transport, and signaling at the time of maternal recognition of pregnancy in cattle: evidence of polycrine actions of prostaglandin E2. Journal of Endocrinology, v.145, p.5280-5293, 2004.

BINELLI, M.; THATCHER, W.W.; MATTOS, R. et al. Antiluteolytic strategies to improve fertility in cattle. Theriogenology, v.56, p.1451-1463, 2001.

BULLMAN, D.C.; LAMMING, G.E. Milk progesterone levels in relation to conception, repeat breeding and factors influencing acyclicity in dairy cows. Journal of Reproduction and Fertility, v.54, p.447-458, 1978.

CASTILHO, E.M.; PFEIFER, L.F.M. Influência do momento da aplicação de prostaglandina em protocolos utilizando progestágenos em vacas de corte pós-parto. A Hora Veterinária, p.25-32, 2005 .

COLAZO, M.G.; KASTELIC, J.P.; MAINAR-JAIME, R.C. et al. Resynchronization of previously timed-inseminated beef heifers with progestins. Theriogenology, v.65, p.557-572, 2006.

CORRÊA, E.S.; ANDRADE, P.; EUCLIDES FILHO, K. et al. Avaliação de um sistema de produção de gado de corte. 1. Desempenho reprodutivo. Revista Brasileira de Zootecnia, v.29, n.6, p.2209-2215, 2000.

FERREIRA, M.M.; FERREIRA, A.C.M.; EZEQUIEL, J.M.B. Avaliação econômica da produção de bovinos confinados. Informações Econômicas, v.34, n.7, p.7-20, 2004. 
GARRETT, J.E.; GEISERT, R.D.; ZAVY, M.T. et al. Evidence for maternal regulation of early conceptus growth and development in beef cattle. Journal of Reproduction and Fertility, v.84, p.437-446, 1988 .

GONÇALVES, P.B.D.; FIGUEIREDO, J.R.; FREITAS, V.J.F. Biotécnicas aplicadas à reprodução animal. São Paulo: Livraria Varela, 2001. 340p.

GUILBAULT, L.A.; THATCHER, W.W.; DROST, M. et al. Influence of a physiological infusion of prostaglandin $F_{2} \alpha$ into postpartum cows with partially suppressed endogenous production of prostaglandins. 1. Uterine and ovarian morphological responses. Theriogenology, v.27, p.931-946, 1987.

HANSEN, P.J. Embryonic mortality in cattle from the embryo's perspective. Journal of Animal Science, v.80, p.E33-E44, 2002 (suppl. 2).

KERBLER, T.L.; BUHR, M.M.; JORDAN, L.T. et al. Relationship between maternal plasma progesterone concentration and interferon-tau synthesis by the conceptus in cattle. Theriogenology, v. 47, p.703-714, 1997

KINDAHL, H.; EDQVIST, L.E.; GRANSTROM, E. et al. The release of prostaglandin $\mathrm{F}_{2} \alpha$ as reflected by 15-keto-13,14-dihydroprostaglandin $\mathrm{F}_{2} \alpha$ in the peripheral circulation during normal luteolysis in heifers. Prostaglandins, v.11, p.871-878, 1976.

LAMMING, G.E.; DARWASH, A.O.; BACK, H.L. Corpus luteum timction in dairy cows and embryo mortality. Journal of Reproduction and Fertility, v.37, p.245-252, 1989.

LOBATO, J.E.P.; ZANOTTA, R.L.D.; PEREIRA, O.A.N. Efeitos das dietas pré e pós-parto de vacas primíparas sobre o desenvolvimento dos bezerros. Revista Brasileira de Zootecnia, v.27, n.5, p.863-867, 1998.

LÓPEZ-GATIUS, F.; LABERNIA, J.; SANTOLARIA, P. et al. Effect of reproductive disorders previous to conception on pregnancy attrition in dairy cows. Theriogenology, v.46, p.643-648, 1996.

MANN, G.E.; LAMMING, G.E. The role of sub-optimal preovulatory estradiol secretion in the aetiology of premature luteolysis during the short oestrus cycle in the cow. Animal Reproduction Science, v.64, p.171-180, 2000.
MANN, G.E.; LAMMING, G.E.; ROBINSON, R.S. et al. The regulation of interferon-tau production and uterine receptors during early pregnancy. Journal of Reproduction and Fertility, v.54, p.317-328, 1999.

MCCRACKEN, J.A.; CUSTER, E.E.; LAMSA, J.C. Luteolysis: a neuroendocrine-mediated event. Physiology Reviews, v.79, p.263-323, 1999.

MERRILL, M.L.; ANSOTEGUI, R.P.; WAMSLEY, N.E. et al. Effects of flunixin meglumine on embryonic loss in stressed beef cows. In: WESTERN SECTION OF ANIMAL SCIENCE, 2003, Miles City. Proceedings... Miles City: American Society of Animal Science, 2003. p.53-55.

NAAZIE, A.; MAKARECHIAN, M.; HUDSON, R.J. Evaluation of life-cycle herd efficiency in cow-calf systems of beef production. Journal of Animal Science, v.77, n.1, p.1-11, 1999.

ODENSVIK, K.; CORT, N.; BASU, S. et al. Effect of flunixin meglumine on prostaglandin F2a synthesis and metabolism in the pig. Journal of Veterinary Pharmacologic Therapeutics, v.12, p.307-311, 1989.

ODENSVIK, K.; GUSTAFSSON, H.; KINDAHL, H. The effect on luteolysis by intensive oral administration of flunixin granules in heifers. Animal Reproduction Science, v.50, p.35-44, 1998.

PETERSON, A.J.; FAIRCLOUGH, R.J.; PAYNE, E. et al. Hormonal changes around bovine luteolysis. Prostaglandins, v.10, p.675-684, 1975 .

PÖTTER, L.; LOBATO, J.F.P.; NETTO, C.G.A.M. Análises econômicas de modelos de produção com novilhas de corte primíparas aos dois, três e quatro anos de idade. Revista Brasileira de Zootecnia, v.29, n.3, p.861-870, 2000.

STATISTIX $^{\circledR}$. Statistix for Windows: user's manual. Tallahassee: 2004. (CD-ROM).

THATCHER, W.W.; BAZER, F.W.; SHARP, D.C. et al. Interrelationships between uterus and conceptus to maintain corpus luteum function in early pregnancy: sheep, cattle, pigs and horses. Journal of Animal Science, v.62, p.25-46, 1986 (suppl. 2).

ZAVY, M.T. Embryonic mortality in cattle. In: ZAVY, M.T.; GEISERT, R.D. (Eds.). Embryonic mortality in domestic species. Boca Raton: CRC Press, 1994. p.99-140 